\title{
Los FabLab y el Design Thinking: nuevas estrategias para el aprendizaje creativo
}

\author{
Luis Mexitli Orozco Torres
}

\begin{abstract}
Resumen
Las nuevas tecnologías de la información están presentes en casi todas las áreas educativas. Éstas pueden ser aplicadas para el desarrollo de nuevos dispositivos, herramientas o productos, que solucionen de manera creativa problemas del entorno sociocultural. Utilizando la metodología de Design Thinking, se logra que jóvenes de bachillerato generen ideas para la solución creativa de problemas de su contexto. Aunado a ello, la implementación de los FabLab en sus escuelas permite a los estudiantes generar soluciones a problemáticas observadas en su entorno. En este trabajo se expone un análisis de la implementación del FabLab en la Escuela Preparatoria Regional de Atotonilco. Los resultados indican que 55\% de los alumnos encuestados muestran la convicción de crear e innovar un proyecto. Asimismo, 52.5\% se enfocan en resolver una problemática social, por lo que la implementación y uso de este tipo de espacios en la educación debe promoverse. De esta manera, por un lado, se facilita la construcción del conocimiento de manera colaborativa y, por el otro, se materializan las ideas creativas que pudieran evolucionar en artefactos beneficiosos para la sociedad.
\end{abstract}

Palabras clave: aprendizaje creativo, FabLab, Design Thinking, innovación en la educación, TIC.

\section{Fablab and Design Thinking: New Strategies for Creative Learning}

\begin{abstract}
New information technologies are present in almost all educational areas. These may result in the development of new devices, tools, or products that solve problems inherent to different sociocultural environments. Taking advantage of the Design Thinking methodology, high school students can generate ideas in order to solve problems relevant to their context in a creative way. In addition, the implementation of FabLab in their schools allows students to generate different solutions to problems in their environment. This article presents an analysis on FabLab implementation at Atotonilco Regional Preparatory School. The results indicate that 55\% of the surveyed students showed the conviction to create and innovate a project. Similarly, 52.5\% of them focused on solving a social problem. In this sense, implementation and use of this sort of spaces for educational purposes should be promoted to facilitate knowledge acquisition in a collaborative way, that also materializes creative ideas that have the potential to contribute to the wellbeing of society.
\end{abstract}

Keywords: creative learning, FabLab, Design Thinking, innovation in education, IcT.

Recepción: 17/12/2018. Aprobación: 30/07/2020. Dol: http://doi.org/10.22201/cuaieed.16076079e.2020.21.6.11 
Luis Mexitli Orozco Torres

Estudió Ingeniería en Comunicaciones y Electrónica, y Maestría en Sistemas de Calidad en la Universidad de Guadalajara. Después, obtuvo una beca para el Doctorado en Educación, Sociedad y Calidad de Vida, en la Universitat de Lleida, en España. Actualmente labora como Profesor Investigador Titular del Departamento de Ciencias Tecnológicas del cucl, en la Universidad de Guadalajara. Es Candidato al Sistema Nacional de Investigadores y responsable del cuerpo académico: Redes de enseñanza-aprendizaje y tecnologías. Sus intereses de investigación son tecnología educativa, evaluación de la calidad de la educación superior y e-learning.

\section{Introducción}

En la actualidad, las nuevas tecnologías de la información están presentes en casi todas las áreas y funciones sociales. Estas herramientas proporcionan bases para innovar y desarrollar soluciones a problemáticas sociales en sus diferentes categorías como la ecológica, psicológica o motriz. Como consecuencia de lo anterior, es necesario adaptarse a los cambios socio-tecnológicos y emplearlos, además de forma creativa, con la intención de solucionar dichas problemáticas. Esta dinámica se logra mediante la aplicación de las tecnologías, al aprovechar la información científica y tecnológica a nuestro alcance.

Por esta razón, es necesario que las nuevas generaciones se alimenten de la curiosidad, que propicia innovaciones en pro del desarrollo de su comunidad y que, además, se complementen con las herramientas que solucionan de manera creativa problemas de su entorno. Para estos efectos, se presenta el Design Thinking como una metodología para generar ideas innovadoras y dar solución a necesidades prioritarias, utilizando de manera creativa y analítica técnicas con un contenido visual predominante y plástico, que resulta en soluciones tanto novedosas como factibles (Magro Gutierrez y Carrascal Domínguez, 2019).

Los pasos en esta metodología varían de cinco a siete, dependiendo del autor. Para Ramos y Wert (2015) se resume en cinco: empatizar, definir, idear, prototipar y probar. Otro ejemplo se puede observar en la imagen 1. A manera de resumen, la metodología integral se explica de la siguiente manera: primero, es necesario descomponer el problema; es decir, dividirlo en partes más pequeñas con el fin de que sea más sencillo su análisis. Después, se plantean en colaboración técnicas como la lluvia de ideas, que permite externar junto a otros miembros del equipo todo tipo de ideas que contribuyan a encontrar alguna solución al problema que se ha planteado (Elsbach y Stigliani, 2018) 
Imagen 1. Desarrollo de una estrategia de Design Thinking. Fuente: Macarrilla, 2017.
Imagen 2. Equipo de FabLab de la Escuela Preparatoria Regional de Atotonilco.

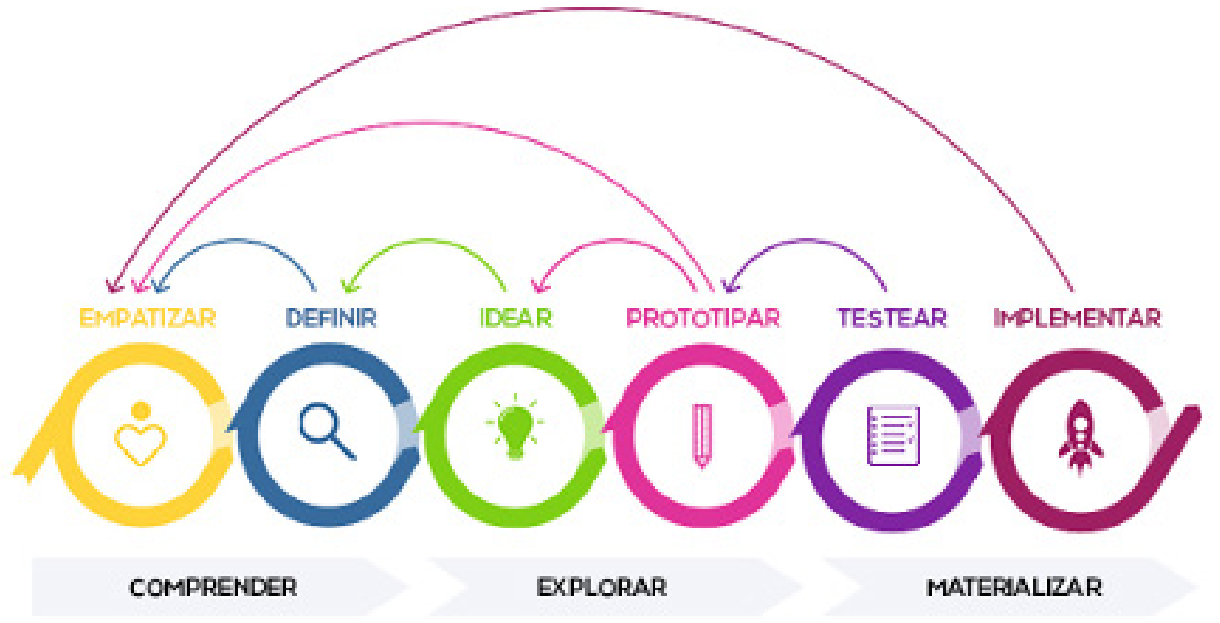

Estos esfuerzos por lograr que jóvenes de bachillerato generen ideas para la solución de problemas de su contexto se materializan con la implementación de los FabLab (Fabulous Laboratories) en las escuelas. Los FabLab son espacios en los que la cultura digital y la creatividad se fusionan con la producción material para lograr una nueva fase en el desarollo educativo (Walter-Herrmann y Büching, 2014). Son una iniciativa del Center of Bits and Atoms (CBA) del Massachusetts Institute of Technology (MIт) para desarrollar laboratorios experimentales utilizando tecnologías de fabricación digital. Estos espacios de colaboración, equipados con máquinas de desarrollo tecnológico, corte láser, router e impresoras 3D, controladas por computadoras para procesos de producción personalizados, pretenden la innovación y desarrollo creativo para la creación de herramientas tecnológicas para solucionar problemas reales (García-Ruiz y Lena-Acebo, 2018).

Por tal motivo, en este trabajo se analiza la implementación de un FabLab en la Escuela Preparatoria Regional de Atotonilco, con la finalidad de verificar si se favorece el desarrollo de los estudiantes a partir de su interacción con este laboratorio y el Design Thinking. 


\section{Desarrollo}

Con la implementación del FabLab en la Escuela Preparatoria Regional de Atotonilco, llegó también el reto, tanto para directivos como para docentes, de generar una dinámica escolar que permita utilizar adecuadamente el laboratorio y así aprovechar al máximo estas instalaciones. Para ello, se decidió generar un curso piloto con grupos elegidos con base en la relación de la asignatura de tecnologías de la información y del análisis de su contexto sociocultural.

Este curso piloto se efectúa en dos etapas principales. En la primera, se realizan talleres en los que los estudiantes conocen herramientas del FabLab y proponen soluciones a problemas de su contexto, utilizando su creatividad e inventiva para desarrollar prototipos que los atenúen o eliminen. Los prototipos se desarrollan con el material a su alcance, con la finalidad de que se recreen en el FabLab mediante el equipo disponible.

En la segunda etapa se recoge la percepción de los estudiantes sobre el trabajo que efectuaron. En esta fase se aplica una metodología de corte cuantitativo, con una encuesta a cuarenta estudiantes involucrados en la dinámica, para así conocer aspectos de motivación y enfoque del prototipo o trabajo que se realizó.

Cabe destacar que un equipo de la Universidad Nacional Autónoma de México realiza una verificación de la implementación con la finalidad de dar un diagnóstico externo a la institución.

A partir de lo que ya se dijo, el plan de ejecución para el desarrollo de la estrategia se puede observar en la tabla 1.

\begin{tabular}{lll}
\multicolumn{1}{c}{ Fecha } & \multicolumn{1}{c}{ Actividades } & \multicolumn{1}{c}{ Recursos } \\
Febrero & Sensibilizar y difundir el proyecto. & Presentación en video. \\
\hline Marzo & $\begin{array}{l}\text { Hacer introducción a la metodología } \\
\text { Design Thinking. }\end{array}$ & Proyector, material impreso. \\
\hline Abril & Inicio de los prototipos. & Materiales personales de los \\
& Cursar el taller de aplicación del & Cortadora láser e impresora 3D. \\
\hline Mayo & FabLab para docentes. & Materiales de los alumnos. \\
\hline Mayo & Revisar los avances de los prototipos. & Presentación. \\
\hline Junio-Julio & Presentación de los prototipos. & Computadora. \\
\hline Agosto & $\begin{array}{l}\text { Aplicar la encuesta de la unAm a } \\
\text { estudiantes y profesores. }\end{array}$ & \\
\hline Septiembre & Visita y uso del FabLab. & Laboratorio FabLab. \\
\hline Octubre & Capacitación del programa que & Computadoras. \\
& controla la cortadora láser &
\end{tabular}

Tabla 1. Plan de ejecución de la implementación del FabLab en la Escuela Preparatoria Regional de Atotonilco. Fuente: elaboración propia.

RDworks V8. 
La necesidad de capacitar en el uso de los FabLab y la aplicación del Design Thinking es de suma importancia, debido a que los profesores deben de familiarizarse primero con lo que supone una método innovador, su finalidad, metodología y aplicación. Además, es menester familiarizarse con los equipos que se usan en el FabLab, el software, así como las características y alcances que tienen los dispositivos. Lo anterior dará una visión global al profesor, para guiar a los estudiantes durante el proceso.

Por otro lado, la revisión de prototipos es importante ya que el profesor debe guiar y recomendar la elaboración de éstos, de acuerdo a parámetros y materiales que están dentro de lo que admite FabLab, así como las dimensiones y características que requieren los equipos que se encuentran en estos laboratorios.

Como resultado principal se obtiene que con la implementación del Design Thinking se generan diferentes soluciones a problemáticas observadas por los estudiantes en su entorno. Estos son realizados en prototipos, elaborados con material a su alcance como cartón, papel, plástico, madera o material reciclado, en general.

Los resultados de la encuesta de percepción de los estudiantes del uso del Fablab se muestran en la imagen 3. De los alumnos, 55\% tiene la convicción de crear e innovar en un proyecto, lo cual es motivante en su formación académica. Por otra parte, $42.5 \%$ desea diseñar y realizar prototipos; sin embargo, un porcentaje mínimo piensa que el FabLab no tiene ninguna aplicación en su vida cotidiana.

\section{PERCEPCIÓN DE ESTUDIANTES DE USO DE FABLAB}

Imagen 3. Resultados de la encuesta de uso que los estudiantes pueden dar al FabLab. Fuente: elaboración propia.

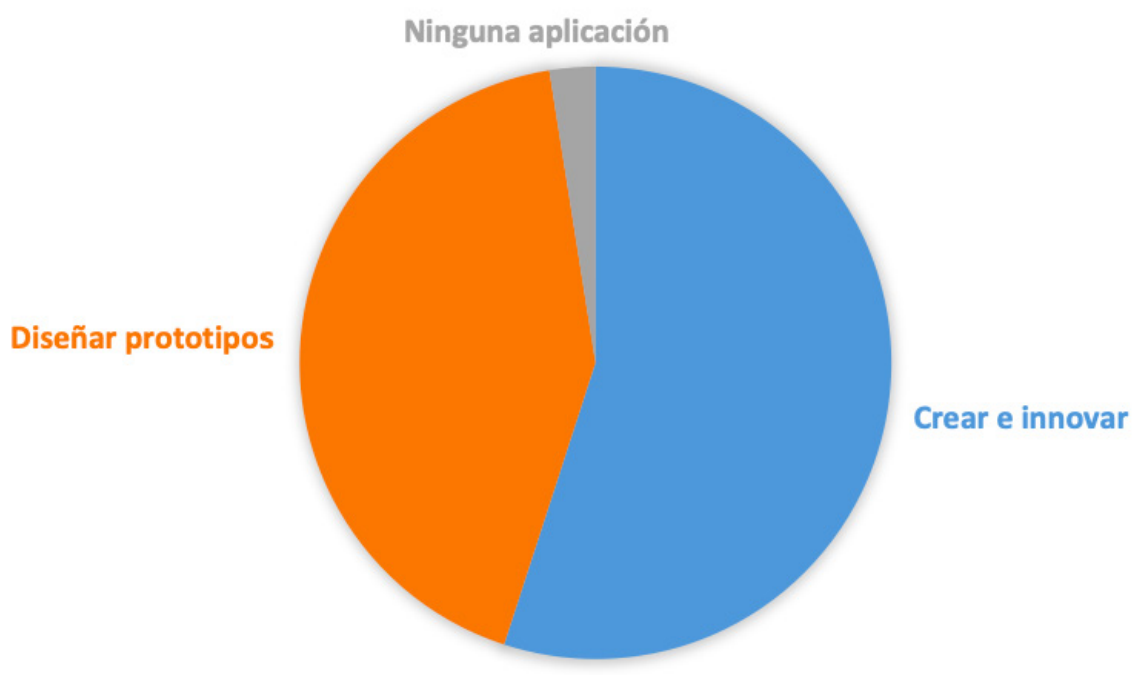

En la imagen 4 se da a conocer el propósito de la participación. 52.5\% de los alumnos se enfoca en resolver una problemática social, 40\% en crear alguna herramienta personal, adaptada alguna necesidad, y un muy bajo porcentaje, de 
Imagen 4. Propósito de creación. Fuente: elaboración propia.

Imagen 5. (Izquierda) Prototipo de generador eléctrico.

Imagen 6. (Derecha) Prototipos de prótesis de mano mecanizada.
7.5\%, sólo tiene la intención de cumplir con el requisito. Las cifras presentadas en ambos gráficos sugieren que los estudiantes se sienten motivados $y$, hasta cierto punto, retados a generar ideas que tengan una aplicación en su entorno.

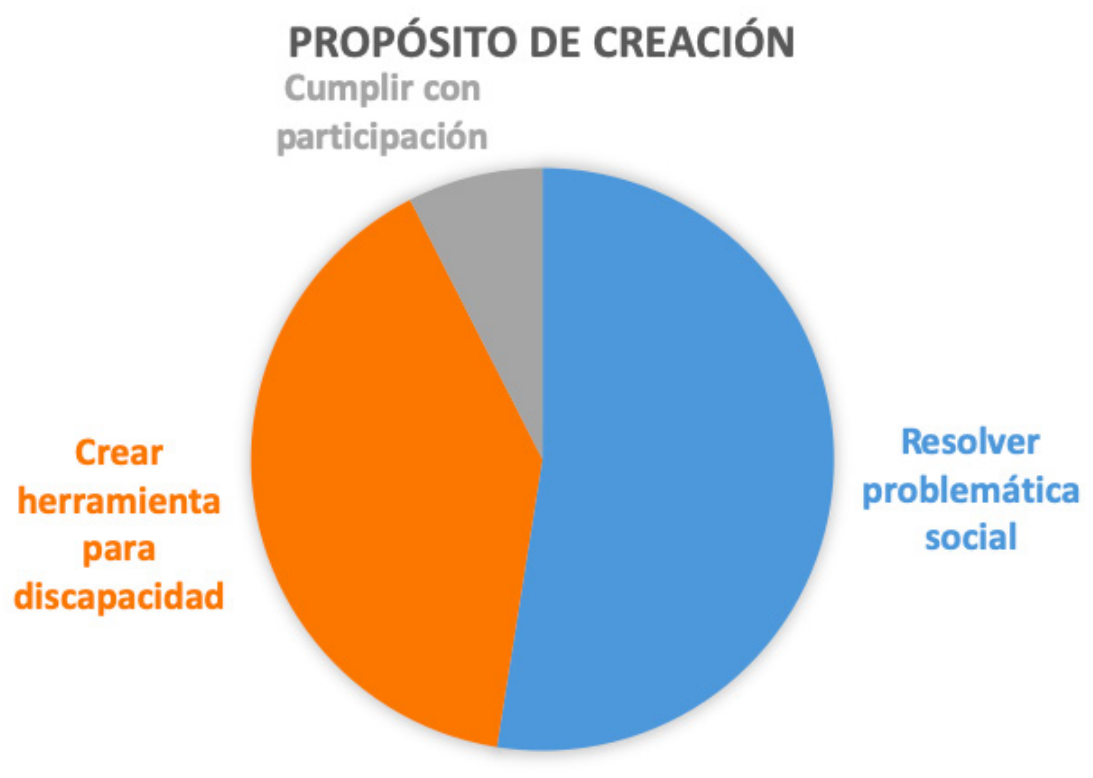

En la imagen 5 se muestra un prototipo de un minigenerador eléctrico, elaborado con material al alcance de los estudiantes. En la imagen 6 se observa un prototipo de una mano mecanizada, la cual podría funcionar como prótesis para personas con discapacidad.
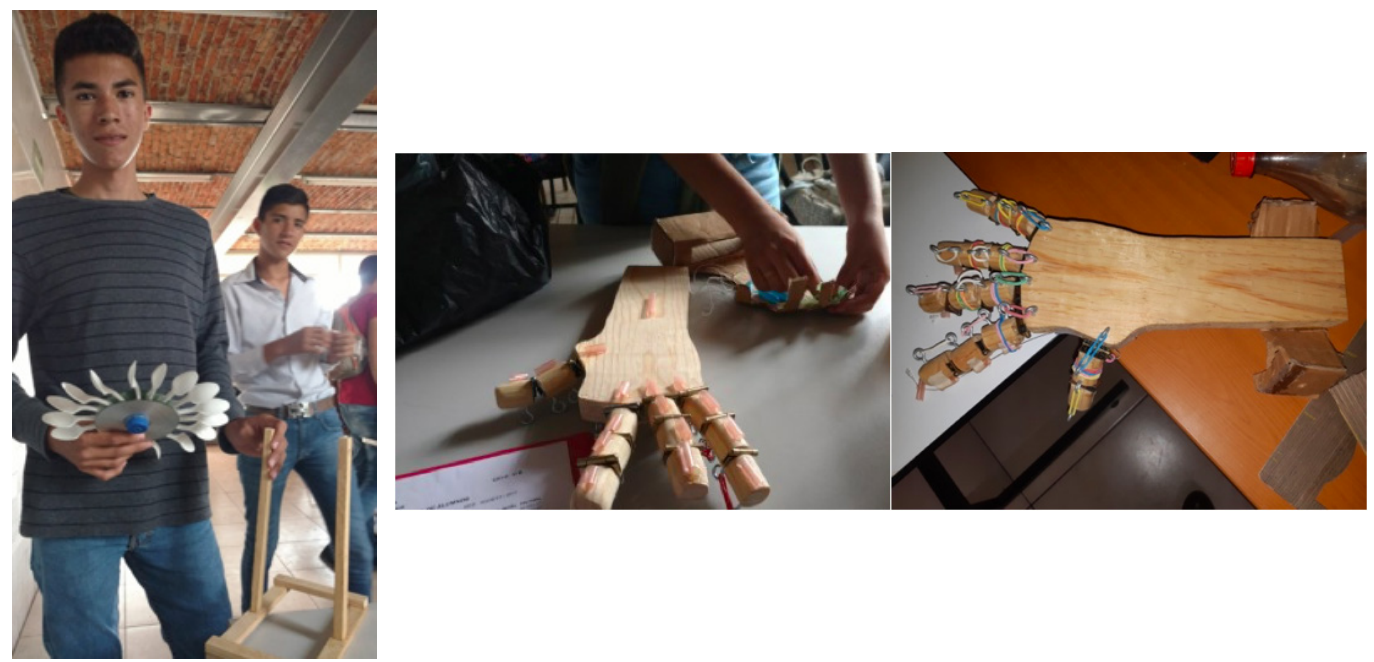

\section{Conclusiones}

El desarrollo y materialización de las ideas con la ayuda del Design Thinking en la etapa educativa temprana es de suma importancia para el crecimiento de los estudiantes (Noel y Liub, 2017). Los alumnos desarrollan, sino es que mejoran, 
habilidades tecnológicas creativas innovadoras, aparte de que se logra un alto nivel de involucramiento en sus proyectos (Acebo, 2017).

En este sentido, el uso de este tipo herramientas en el futuro de la educación debe promoverse, porque facilita la construcción del conocimiento de manera colaborativa y despierta la inquietud en los estudiantes, al materializar ideas en artefactos que son útiles a la sociedad (Walter-Herrmann y Büching, 2014).

Esto apunta a que la implementación del FabLab en la Escuela Preparatoria Regional de Atotonilco favorece el desarrollo de los estudiantes a partir de su interacción con el FabLab y el Design Thinking. Por lo tanto, el programa debe extenderse a todos los grados y grupos del plantel, bajo la premisa de un desarrollo continuo de ideas conforme el estudiante avanza en sus estudios de bachillerato, para concretar un prototipo final al culminar su grado.

De igual manera, es necesario proyectar y construir espacios como el FabLab en las escuelas donde los alumnos puedan ser más creativos y desarrollen habilidades para el uso de la tecnología aplicada en ideas innovadoras. Esto, en el entendido de que hay proyectos que evolucionan con el estudiante a lo largo de su vida académica y personal, $y$, al final, dichas ideas tienen el potencial de convertirse en nuevas tecnologías, herramientas o patentes en beneficio de la sociedad y su entorno.

\section{Referencias}

* Acebo, F. J. L. (2017). Ecologías colaborativas: análisis y caracterización del fenómeno FabLab [tesis de doctorado, Universidad de Cantabria].

* Elsbach, K. D., y Stigliani, I. (2018). Design thinking and organizational culture: A review and framework for future research. Journal of Management, 44(6), 22742306. Dol: https://doi.org/10.1177/0149206317744252

* García-Ruiz, M. E. y Lena-Acebo, F. J. (2018, junio). FabLab global survey: Characterization of FabLab phenomenon. 2018 13th Iberian Conference on Information Systems and Technologies, pp. 1-6. Dol: https://doi.org/10.23919/ CISTI.2018.8399154

- Macarrilla, C. (2017). Design Thinking [infografía que describe el proceso de Design Thinking]. https://commons.wikimedia.org/wiki/File:Designthinking.png\#filelinks

* Magro Gutierrez, M. y Carrascal Domínguez, S. (2019). El Design Thinking como recurso y metodología para la alfabetización visual y el aprendizaje en preescolares de escuelas multigrado de México. Vivat Academia (146), 71-95. Dol: https://doi. org/10.15178/va.2019.146.71-95

* Noel, L. Ay Liub, T. L. (2017, mayo). Using Design Thinking to Create a New Education Paradigm for Elementary Level Children for Higher Student Engagement and Success. Design and Technology Education: an International Journal, 22(1). https:// ojs.Iboro.ac.uk/DATE/article/view/2198 
* Ramos, R., y Wert, A. (2015). ¿Qué es el Design Thinking? Design Thinking en español. http://designthinking.es/home/index.php

- Walter-Herrmann, J., y Büching, C. (Eds.). (2014). FabLab: Of machines, makers and inventors. Transcript Verlag.

\section{Cómo CITAR ESTE ARTículo}

Orozco Torres, Luis Mexitli. (2020, noviembre-diciembre). Los FabLab y el Design Thinking: nuevas estrategias para el aprendizaje creativo. Revista Digital Universitaria (RDU), 21(6). Dol: http://doi.org/10.22201/cuaieed.16076079e.2020.21.6.11 